\title{
e-Phaïstos
}

e-Phaïstos Revue d'histoire des techniques / Journal of the history of technology

IX-1 | 2021

Autour de Léonard de Vinci

\section{Les machines de Léonard de Vinci comme vision du monde}

Leonardo da Vinci's Machines as a Vision of the World

\section{Gérard Chazal}

\section{(2) OpenEdition}

Journals

Édition électronique

URL : https://journals.openedition.org/ephaistos/8929

DOI : 10.4000/ephaistos.8929

ISSN : 2552-0741

Éditeur

IHMC - Institut d'histoire moderne et contemporaine (UMR 8066)

Référence électronique

Gérard Chazal, «Les machines de Léonard de Vinci comme vision du monde », e-Phaïstos [En ligne], IX-1 | 2021, mis en ligne le 27 avril 2021, consulté le 16 septembre 2021. URL : http://

journals.openedition.org/ephaistos/8929; DOI : https://doi.org/10.4000/ephaistos.8929

Ce document a été généré automatiquement le 16 septembre 2021.

Tous droits réservés 


\title{
Les machines de Léonard de Vinci comme vision du monde
}

\author{
Leonardo da Vinci's Machines as a Vision of the World
}

\author{
Gérard Chazal
}

1 Dans son ouvrage consacré aux ingénieurs de la Renaissance, Bertrand Gille donne une critique assez sévère de l'œuvre de Léonard de Vinci quant à la représentation de machines (Gille 1964). Pour B. Gille, la plupart des machines de Léonard de Vinci sont des copies de celles proposées par d'autres ingénieurs qui l'ont précédé comme Francisco di Giorgio Martini, Jacomo Fontana ou Taccola ou des dessins de machines déjà existantes qu'il a pu observer. Même les machines qui n'ont pas été réalisées ou qui ne sont pas réalisables dans l'état des techniques à l'époque, sont pour B. Gille présentes chez les prédécesseurs. Il écrit ainsi :

\begin{abstract}
« Mis à part la machine volante, quelques machines textiles, la machine à polir les miroirs, tout le reste est emprunté, souvent directement emprunté à ses devanciers » (Gille 1964 : 153).
\end{abstract}

2 Si l'on veut bien accepter ce que dit Bertrand Gille comme effectivement vérifié, il n'en demeure pas moins que cette vision laisse échapper deux choses essentielles dans l'approche des machines par Léonard de Vinci.

3 Tout d'abord, ces remarques ne prennent pas en compte la magie des dessins de Léonard où se mêlent une forme de rationalité technique et la puissance de l'imaginaire. La raison et l'imagination sont étroitement enchevêtrées dans les Carnets qui ne sont pas une œuvre technologique proprement dite ni une œuvre plastique au sens propre; ce sont le lieu où se posent ses pensées, ses observations et ses rêves. D'où des machines irréelles ou irréalisables, machines rêvées autant que conçues à côté de machines déjà existantes.

4 Ensuite, ce qui peut paraître essentiel, c'est que les machines dessinées, décomposées et recomposées, mêlées à des dessins d'écoulements de fluides et surtout d'anatomie, participent d'une véritable vision du monde, à la fois nature et artifice. Il n'est pas sûr 
que le projet technique, tel qu'il apparaît dans les dessins de Léonard de Vinci, vise nécessairement une réalisation - c'est pourquoi il y a toujours eu quelque chose d'un peu vain dans l'entreprise de construction de maquettes selon les présentations de machines dans les Carnets - il manifeste bien souvent une appropriation du monde sur un mode poétique autant que technique, par entrelacement des deux. Les Carnets ainsi que les différents manuscrits ne sont pas des œuvres destinées à une publication ou des plans pour des projets précis mais des notes dans lesquelles Léonard de Vinci a pu dessiner légitimement autant ce qu'il pouvait voir dans la réalité ou dans d'autres ouvrages que ce qu'il imaginait. De ce fait l'accusation d'emprunt de Bertrand Gille tombe d'elle-même. C'est ce qu'il s'agit de montrer dans ce texte.

\section{L'expression graphique de la possibilité d'une maîtrise du monde}

5 Léonard de Vinci, né en 1452 et mort en 1519, appartient à une période où le vieux monde étroitement limité à l'Europe, l'Afrique du Nord et les portes de l'Asie, centré sur Rome ou Jérusalem, craque. C'est le temps où l'Europe va découvrir le monde et en entreprendre la conquête. Ainsi Léonard de Vinci a 36 ans en 1488 quand Bartolomeu Dias passe le Cap de Bonne Espérance ; il a 40 ans en 1492 lorsque Christophe Colomb découvre l'Amérique; en 1497, il a 45 ans lorsque Jean Cabot explore les côtes d'Amérique du Nord et 48 ans en 1500, alors que Vasco de Gama débarque au Brésil. Magellan contournera l'Amérique ouvrant la route du Pacifique l'année de sa mort, en 1519. Difficile de dire ce que Léonard savait de ces différents voyages mais on perçoit très bien à travers ces quelques dates que le monde s'ouvre et se livre à la conquête. Cela est vrai de l'espace, à l'échelle d'une vie, à l'échelle de la planète. Cela est aussi vrai à l'échelle des tâches de maîtrise des choses, maîtrise des eaux, maîtrise du ciel. Cela vaut encore pour la maîtrise du vrai et du beau, du corps et de l'artifice. À l'exploration des mers et des océans, des terres immenses correspond celle des anatomies, des mouvements les plus fins, des gestes et de leurs équivalents mécanisés, de l'embryogenèse (dessins de fœtus dans le ventre maternel, voir fig.1), des écoulements de fluides, du vol des oiseaux, de la marche animal et humaine, des effets de l'âge ou de l'enchaînement des roues dentées constituant des machines. 
Fig.1. Fœtus dans l'utérus

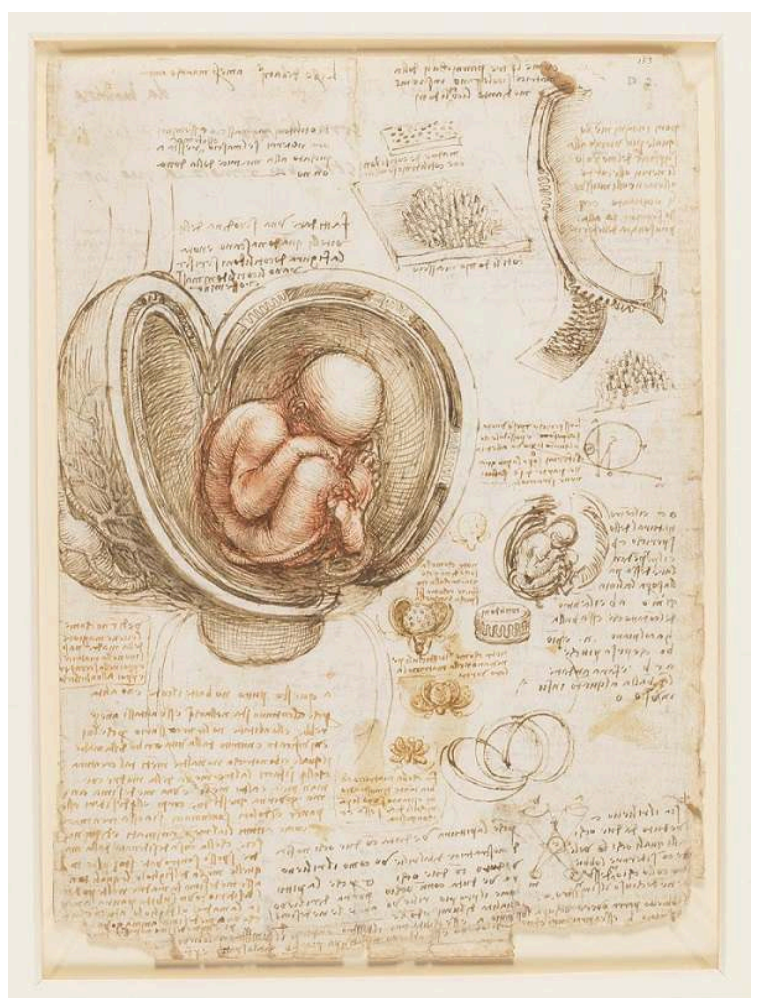

L'exploration du monde qu'entreprenaient les hommes de la Renaissance ne vise pas seulement le dévoilement de nouvelles terres, l'ouverture de nouveaux horizons, mais s'attache aussi à tout ce qui demeure caché dans la nature la plus proche ou comporte une part de mystère, comme le développement de la vie dans l'utérus. Les dessins d'anatomie de Léonard de Vinci font écho, d'une certaine manière aux travaux de Vésale.

Royal Collection Trust/@ Her Majesty Queen Elisabeth II 2021

6 À cette perspective d'une maitrise du monde en train de s'élargir correspond, chez Léonard de Vinci, la mise en œuvre d'une méthode que l'on pourrait résumer par analyse-décomposition, synthèse-reconstruction et usage d'outils abstraits.

7 Les deux premiers aspects méthodologiques sont particulièrement illustrés par ces engrenages dessinés d'abord en éléments séparés, puis regroupés dans un ensemble efficient (Codex Atlanticus f.8v. ainsi que dans Codex de Madrid I. f.29v. et f.30r.) ou encore dans l'étude de mécanismes d'horlogerie (Codex Atlanticus f.348v., f.399v. et Codex de Madrid I. f.45r., f.36v., f.8v., f.14r., f.27r. $)^{1}$. Dans chaque cas, le dispositif global est accompagné de certains de ses éléments pris séparément. On retrouvera le même jeu de décomposition et recomposition dans les études d'anatomie où il s'agit de fournir une représentation de la machine organique. Cela est particulièrement visible dans les 215 planches d'anatomie conservées dans la Bibliothèque Royale de Windsor. Le dessin va suivre une marche de l'aspect extérieur vers les rouages internes comme s'il s'agissait de décomposer sous notre regard les différents organes d'une machinerie complexe, de suivre les étapes d'une dissection ou d'un véritable « démontage » (fig.2). 
Fig.2. Anatomie de l'épaule et du cou

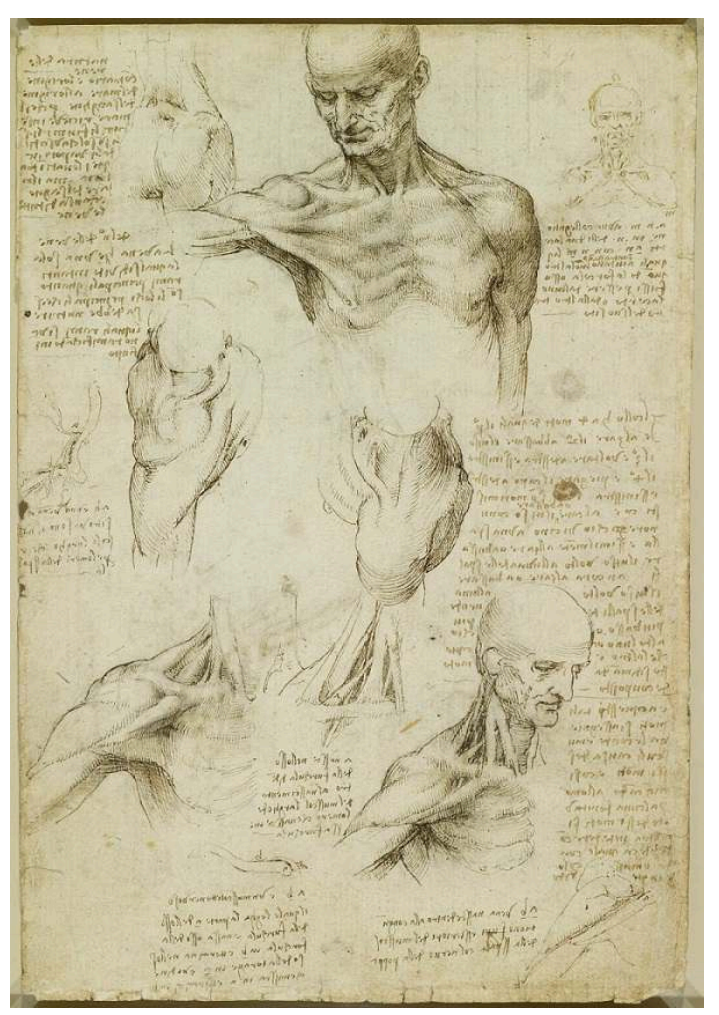

Bien sûr l'étude de l'anatomie correspond à ce désir de mieux connaître l'intérieur des corps pour mieux en représenter l'extérieur, pour mieux rendre la dynamique du mouvement dans la peinture et la sculpture. Mais ce travail de Léonard de Vinci noté probablement à la table de dissection suppose une conception du corps proche de celle d'une machine dont la dynamique repose sur un certain arrangement des éléments articulés entre eux. Ce « mécanisme » n'est d'ailleurs pas incompatible avec une forme de vitalisme dans la mesure où l'on peut étudier l'organisation mécanique en mettant entre parenthèses la force motrice.

Royal Collection Trust/@ Her Majesty Queen Elisabeth II 2021

D'abord les membres tels que nous pouvons les apercevoir, puis les muscles mis à nu, le squelette et le jeu des articulations, les organes internes en leurs places respectives, jusqu'au fœetus dans l'utérus. On sait que Léonard de Vinci a assisté à des dissections. Représenter le corps, c'est en suggérer la maitrise, peut-être le soin, comme la représentation des mécanismes d'horlogerie évoque la maîtrise du temps, ou comme encore le dessin des écoulements de fluide, en particulier des eaux tumultueuses, en prépare le domptage par des dispositifs hydrauliques.

9 La machine organique fait écho à l'artifice, la machine construite de bois et de métal. Le vol des oiseaux, l'anatomie de l'aile vient se refléter dans les dessins de machines volantes (Codex sur le vol des oiseaux, Bibliothèque de Turin, ainsi que les différents dessins de l'ornitottero dans le manuscrit B de Paris, f.75r., f.79r., f.80v., f.89r. et dans le Codex Atlanticus f.313v.-a et f.302v.-a)). Certes, Léonard de Vinci n'est pas ce que sera plus tard La Mettrie, un matérialiste mécaniste, mais, chez lui, la maîtrise du monde qui commence par la connaissance que l'on en peut avoir, suppose le même type d'analyse par la représentation que celle qui est à l'œuvre dans l'invention des machines, celles qu'il a pu rencontrer comme celle dont il est le créateur, celles qui sont réalisables ou qui furent réalisées comme celles qui, irréalisables, tiennent du rêve d'un monde qui livre ses lois et, par-là, se livre à notre pouvoir. 
Cependant, et Léonard de Vinci en est parfaitement conscient, le dessin appelle le calcul tout autant que l'abstraction géométrique. On sait qu'il s'est fait l'élève du mathématicien Luca Pacioli. Il est vrai que la Renaissance est le siècle de l'introduction de la géométrie dans la représentation, avec la découverte de la perspective, mais aussi les diverses constructions géométriques que vont utiliser les peintres, les architectes et les ingénieurs. Les mathématiques pratiques que met en œuvre Léonard de Vinci relèvent de la construction abstraite des formes au même titre que les dessins de machines relevaient de la construction d'objets matériels et concrets. Cet aspect constructif des mathématiques apparaît clairement dans le manuscrit A de l'Institut de France à Paris (Paris Manuscrit A f.11v. et A f.12r. en particulier où il s'agit du tracé de polygones et de la division de la circonférence et du carré par construction géométrique).

11 D'une manière générale, l'usage des mathématiques chez Léonard de Vinci, qu'il s'agisse de constructions géométriques utiles pour le tracé de différentes pièces mécaniques ou de calcul comme lorsqu'il s'agit d'établir le rapport entre la surface de l'aile (de l'oiseau ou artificielle) et le poids porté, lui permet de dépasser la simple observation, les expériences concrètes et un empirisme immédiat vers des considérations générales; dans les dessins, le particulier est subsumé sous le général. Le particulier ne disparaît pas puisque chaque dessin demeure singulier, mais le passage au général se fait par la multiplication des décompositions et des points de vue. Un exemple significatif de cette méthode est probablement l'invention du changement de vitesse (Codex Atlanticus f.27v.-a). Cette dialectique "graphique» du particulier et du général prend bien évidemment toute sa signification dans le célèbre " homme de Vitruve " où viennent s'illustrer à travers à la fois un corps particulier et les formes abstraites du carré et du cercle une sorte d'idéal de la forme humaine.

Ainsi, qu'il s'agisse de décrire la nature, d'assister à des dissections, de s'instruire en mathématiques et surtout de mettre ces connaissances au service de la création d'un monde d'artifices mécaniques mi réels, mi imaginaires, la maîtrise du monde commençait par une insatiable curiosité et un irrépressible besoin d'agir sur les choses en créant les outils de cette activité à travers les jeux de la représentation.

Bien sûr, les textes de Léonard de Vinci ont leur importance et souvent éclairent les dessins plus que les dessins n'éclairent le texte. Il y a donc au cœur de la méthode comme une forme de primat du dessin. Dès lors, il faut peut-être s'arrêter non seulement sur les formes mais aussi sur la mise en scène des machines à laquelle se livre Léonard de Vinci. Certes, les dessins que l'on trouve dans les Carnets sont, en quelque sorte, des notes graphiques et non des œuvres achevées et peuvent donc apparaitre dépourvus de toute organisation (fig.3). La plupart des machines sont sur fond neutre, en vue d'ensemble ou en vue éclatée. Cependant, elles entrent aussi parfois dans une mise en scène où une silhouette humaine intervient comme s'il s'agissait de mettre en évidence le jeu de maîtrise sur les choses que la machine accorde. Car les machines de Léonard de Vinci appellent la présence d'un agent humain et c'est même, dans certains cas, cet agent qui donne sens à la machine en explicitant sa fonction. Ainsi le célèbre parachute du Codex Atlanticus (f. 381v.-a) soutient un homme silhouetté. Dans le même Codex (f. 276r.-b) la machine volante porte un être humain. Toujours dans le Codex Atlanticus, la machine pour marcher sur l'eau (f. $7 \mathrm{v}$.), l'arbalète géante (f. 53v.) ainsi que beaucoup de machines de guerre représentent des hommes s'en servant ou les servant (fig.3 et 4). 
Fig.3. Armes et chars de combat

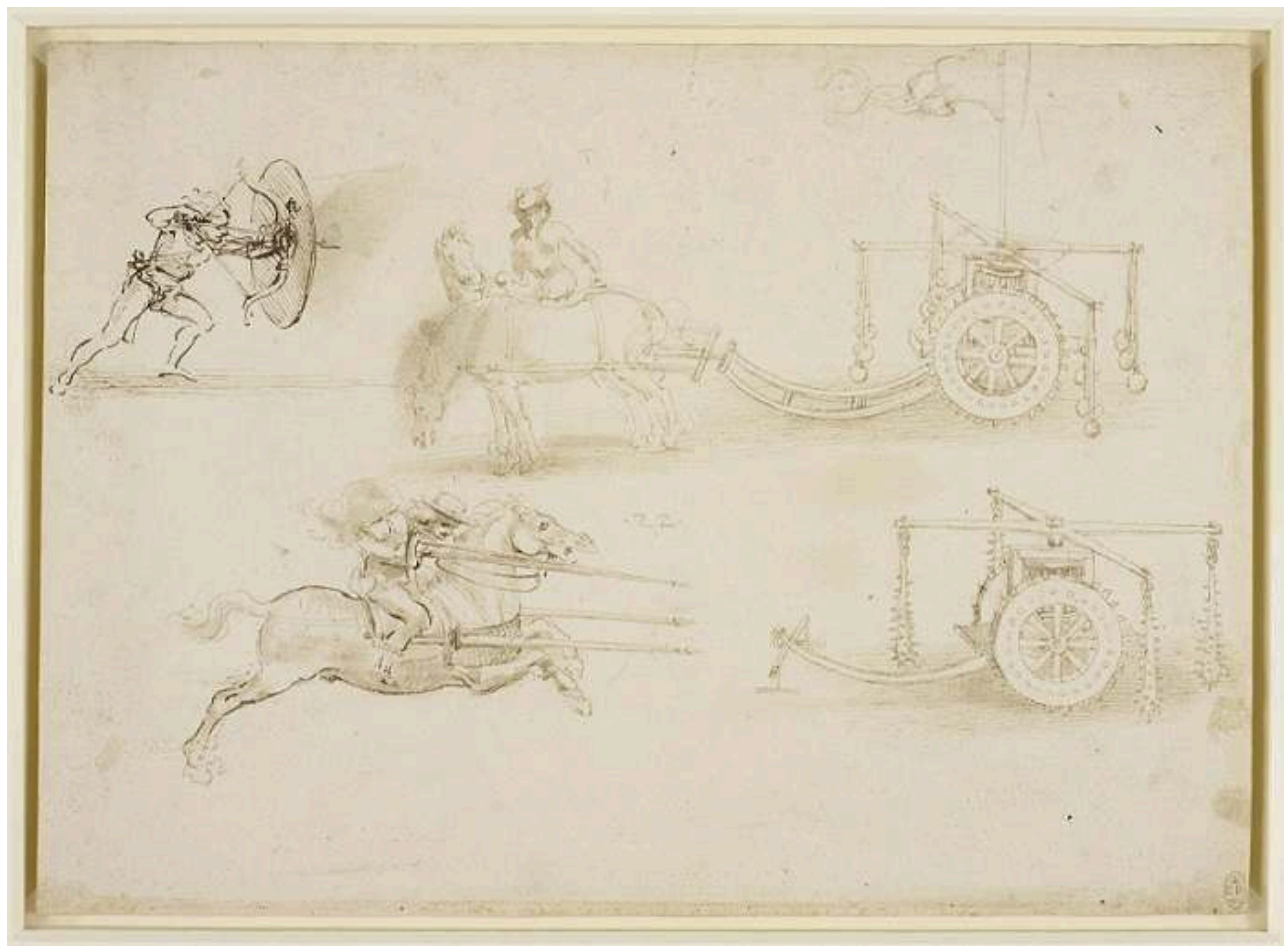

L'époque se réfléchit nécessairement dans l'œuvre des artistes et surtout des ingénieurs. Comme beaucoup d'autres ingénieurs de la Renaissance, Léonard de Vinci est intéressé par les problèmes de l'armement, non seulement parce que des recherches dans ce sens sont imposées par les commanditaires qui font vivre, mais parce que la question des armes soulève des problèmes techniques particulièrement intéressants.

Royal Collection Trust/@ Her Majesty Queen Elisabeth II 2021

Tout se passe, dans les représentations de Léonard de Vinci comme si les corps humains venaient s'introduire dans le dispositif machinique en tant qu'éléments ou que la machine se déployait comme prolongement du corps. Il est tout à fait remarquable que, bien souvent, les personnages humains mis en prise avec des machines soient nus comme, par exemple, dans le dessin d'une machine permettant de soulever un énorme canon de la bibliothèque de Windsor (n. 12647). Cela ne va pas sans rappeler les figures d'anatomies qui exposent les parties du corps humain comme les éléments d'une machine. La complexité anatomique se reflète dans la complexité mécanique, dans une mise en scène dynamique. Pour le dessin de la machine soulevant un canon, les membres des nombreux personnages deviennent autant de leviers de la machine. L'organique et le mécanique se superposent et s'enchevêtrent. Les corps se penchent au rythme de la charpente monstrueuse, se confondent avec elle. Quand le corps humain est gagné par une forme de mécanisme, la machine prend des allures organiques. On retrouve le même type de mise en scène de personnages humains autour d'une machine dans le dessin représentant un char armé de faux tournantes, conservé à la bibliothèque de Turin. Mais, là, aux chevaux et à leur conducteur s'opposent les corps mutilés par la machine. La machine se mêle à la vie mais aussi à la mort. 


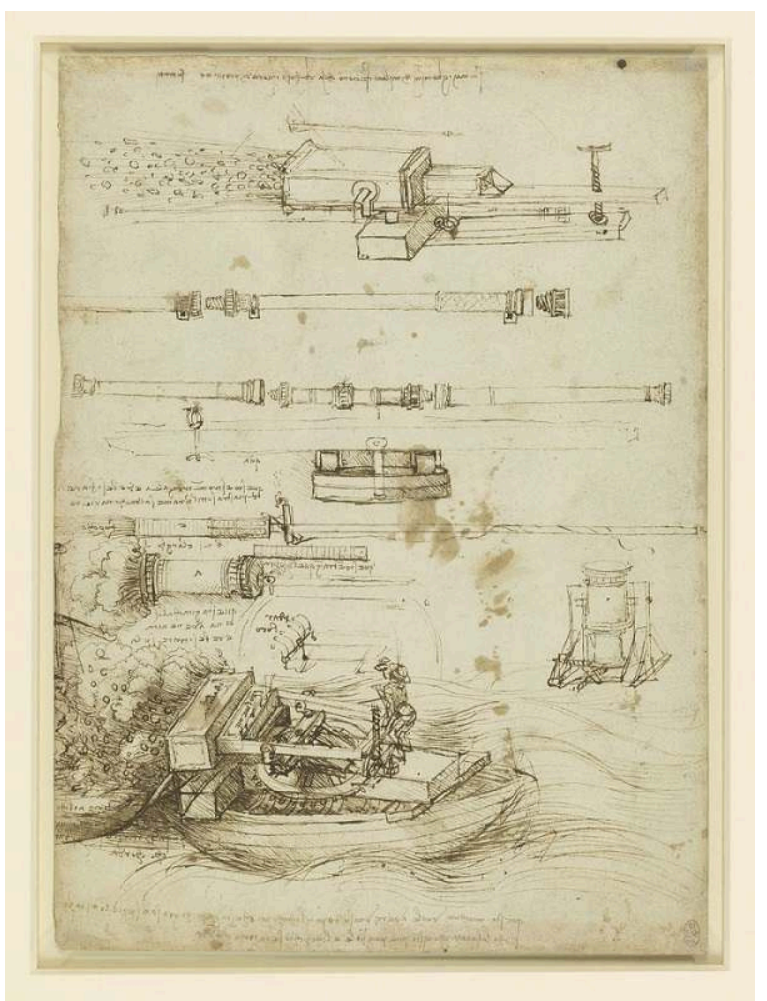

Voici encore un certain nombre de dessins d'armes (canons et mortiers) probablement destinées à envoyer un feu grégeois sur des positions ou navires ennemis pour les incendier. Lorsque Léonard de Vinci arrive à Milan, il y trouve une importante « industrie » d'armement. D'où probablement son intérêt pour ce sujet, même si, comme cela a été déjà écrit, certaines de ces armes ne pouvaient pas fonctionner. Ainsi du bateau équipé de mortiers dont le recul provoquerait certainement le naufrage. Mais il faut peut-être voir là la manière dont Léonard de Vinci met son imagination au service du monde dans lequel il évolue.

Royal Collection Trust/@ Her Majesty Queen Elisabeth II 2021

\section{Une vision mécaniste mais dynamique : la machine entre un mécanisme cosmologique et un mécanisme organique}

Les historiens de la technique ont parfois tendance à isoler les machines dans l'œuvre de Léonard de Vinci, tout du moins dans ses carnets et dans les nombreux dessins conservés à Florence, Turin et Venise. On distinguerait ainsi de grandes catégories : à côté des machines, il y aurait les dessins d'anatomie, les représentations de tourbillons, les constructions géométriques, les œuvres préparatoires à des tableaux... Ce découpage laisse alors échapper l'unité de l'œuvre dessinée de Léonard de Vinci. Et cette unité tient dans la recherche d'une reproduction des dynamismes qui habitent le monde naturel, aussi bien la matière inerte que la matière vivante.

En effet, pour Léonard de Vinci, il s'agit de saisir les flux : ceux qui animent le monde des fluides, de l'écoulement des eaux aux mouvements des fumées et de l'air autour des ailes artificielles. Le plus évanescent prend comme une espèce d'épaisseur : ainsi la vis aérienne, ancêtre possible de l'hélicoptère (Manuscrit de Paris, B f.83v.) pénètre l'air par le mouvement tournant de ses pales. Le mouvement, qu'il s'agisse de celui l'eau (fig. 
5), des nuages de l'orage ou d'un déluge tel qu'il apparaît dans plusieurs dessins des manuscrits de la bibliothèque de Windsor, du vol des oiseaux (Codex sur le vol des oiseaux de Turin) ou des chevaux dans de nombreux dessins préparatoires à des peintures ou de celui décomposé et recomposé des corps humains et des machines, le mouvement donc trace dans l'espace le temps qui dure. Ce sont ces traces que recueillent les dessins de Léonard de Vinci. À cet égard est significatif le dessin 12579r. des manuscrits de la bibliothèque de Windsor qui représente un vieillard assis face à des tracés d'écoulements tumultueux d'eaux. Le temps s'écoule avec tous ses remous et vient laisser sa trace sur le visage et le corps de l'homme dont la vie s'écoule à l'égal des éléments naturels : tel pourrait être le sens de ce rapprochement entre le vieillard et le flux des eaux.

\section{Fig.5. Écoulement tourbillonnaire}

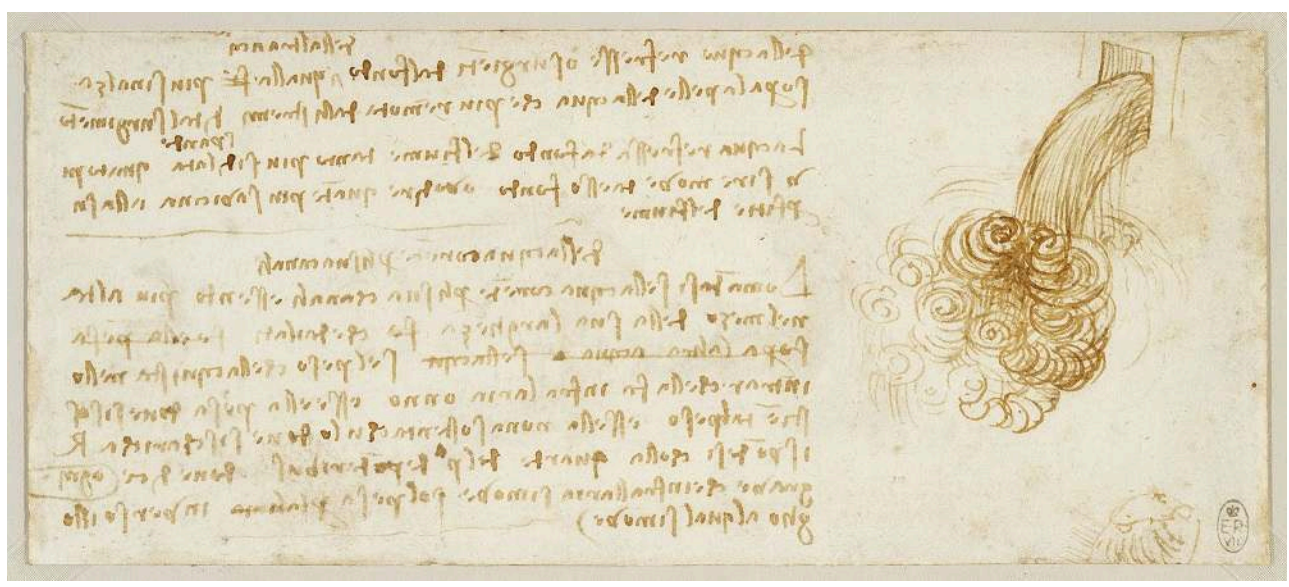

Dans l'univers de Léonard de Vinci tout est mouvement. II y a bien sûr les mouvements provoqués, organisés, ceux du corps humain et ceux de la machine mais il y a aussi les mouvements de la nature, ceux des fluides, des fumées et surtout des eaux qui semblent avoir fasciné l'ingénieur. Car, finalement, tout mouvement révèle des forces naturelles que l'on doit canaliser pour, au bout du compte, les mettre au service de l'art du constructeur de machines ou de l'architecte.

Royal Collection Trust/@ Her Majesty Queen Elisabeth II 2021

Il semble que le monde de Léonard de Vinci soit une vaste mécanique en mouvement qui dépasse et englobe aussi bien les éléments entraînés dans des courants plus ou moins enchevêtrés que le vivant dans son jaillissement. Cependant cette mécanique a quelque chose de cosmologique en même temps qu'elle comporte les échos d'une forme de vitalisme propre à la Renaissance. Il y a en effet quelque chose d'organique dans ce mécanisme, ce que souligne le rapprochement entre les dessins de machines et ceux d'êtres vivants ou d'anatomie animale et humaine. Si les eaux prennent souvent des allures de bêtes furieuses, si elles se tordent comme se tord le corps d'un cheval dont le galop est réfréné, c'est qu'elles participent de cette dynamique organique. Elles ont, comme les nuages ou certaines esquisses de paysages, quelque chose de vivant alors même que les gravures d'anatomie mécanisent des êtres vivants en particulier humains.

Les dessins, en présentant ces tourbillons, ces amoncellements, ces mouvements des choses, en dévoilent une sorte de vie parfois latente, parfois déchainée. Inversement, ils découvrent ailleurs le mécanisme sous-jacent aux organismes vivants. On a dit que les dessins d'anatomie de Léonard de Vinci relevaient de l'acquisition d'une connaissance nécessaire à la juste représentation des corps plus ou moins idéalisés dans la peinture. 
On aurait là le début de ce que vont devenir les cours d'anatomie dans les écoles des Beaux-Arts. Connaître les structures internes pour mieux représenter l'apparence extérieure, il y a certainement de cela dans les travaux de Léonard de Vinci mais il y a peut-être aussi autre chose. Il s'agit notamment de s'interroger par une description anatomique sur le dynamisme de la vie, sur la manière dont les forces façonnent des formes. Cela est particulièrement perceptible dans les dessins de musculatures telles qu'elles apparaissent, par exemple, dans les manuscrits de la bibliothèque de Windsor, exploration minutieuse des machineries organiques.

Dès lors, les machines apparaissent comme des intermédiaires entre le vivant et l'inerte, non pas seulement parce qu'elles nous permettent d'intervenir sur le monde avec plus de force (d'où les nombreuses grues, treuils, palans...), avec plus de rapidité et de précision (la machine à polir les miroirs (Codex Atlanticus f.396v.) ou celle à tailler les limes (Codex Atlanticus f.6r.-b), par exemple), mais parce qu'elles reprennent, sur des modes différents, les gestes-mêmes du vivant, parce qu'elles simulent l'animal ou l'homme. Cette simulation pourra aller jusqu'à une sorte d'automatisme. Il en est ainsi du tournebroche automatique (Codex Atlanticus f.5v.) dont le fonctionnement est assuré par le mouvement de l'air chaud s'élevant du feu dans la cheminée via une hélice. Ce mouvement d'élévation de l'air chaud assure une rotation de la broche d'autant plus rapide que le feu est fort, évitant ainsi qu'une rotation trop lente occasionne une cuisson trop forte et mal répartie de la pièce mise à cuire. Nous avons là une forme de machine automatique qui vient se placer entre le baille-blé des moulins médiévaux et le régulateur à boules de Watt qui viendra plus tard. Encore une fois, tout se passe comme si l'autonomie du vivant venait se réfléchir dans l'organisation mécanique des machines et l'inspirer et, inversement, comme si la structure des machines éclairait celle des organismes.

En fait, l'œuvre de Léonard de Vinci entrecroise graphiquement les forces naturelles, flux et tourbillons, les mécanismes de toutes sortes d'artifices et les formes du vivant. Ces trois domaines ne se confondent pas - il n'y a pas, par exemple, d'andrö̈des ou d'équivalent du Golem chez Léonard de Vinci - mais ils se répondent, entrent en résonance et finalement se comprennent les uns par les autres.

\section{Conclusion}

21 L'œuvre de Léonard de Vinci appartient bien, en ce qui concerne les machines, celles qu'il a vues comme celles qu'il a lui-même conçues, au champ de l'invention technique - ce qui ressort malgré tout de l'analyse de Bertrand Gille - mais elle dépasse très largement ce domaine. Elle est en quelque sorte une "pensée graphique » du monde, une pensée de son temps, le temps d'un monde qui finit et d'un autre qui émerge, celui des conquêtes de la planète par les puissances européennes. Et l'image du monde qui émerge de ce parcours que l'on peut faire à travers les Carnets est celle d'un monde dont l'homme doit acquérir la maîtrise par le savoir, celui de la médecine et celui des mathématiques, autant que par le savoir-faire à travers les objets techniques. En ce sens, Léonard de Vinci préfigure Descartes et son injonction à nous rendre "comme maitres et possesseurs de la nature». Cependant, contrairement à Descartes, le mécanisme n'a pas encore réduit l'organique chez Léonard; celui-ci demeure dans les dessins des forces naturelles et pénètre les machines de présence humaine. Il reste que 
là, dans cette "pensée graphique", sur un mode encore largement imaginaire, s'ouvrent les portes de la modernité.

\section{BIBLIOGRAPHIE}

GILLE Bertrand, Les ingénieurs de la Renaissance, Paris, Hermann, 1964

PEDRETTI Carlo (coordination et introduction), Leonardo da Vinci, The Complete Works, Newton Abbot, Devon, UK, David and Charles Publishers, 2006

CIANCHI Marco, Les machines de Léonard de Vinci, Florence, Ed. Becocci, 1984

\section{NOTES}

1. Pour les références aux dessins de Léonard de Vinci, on utilise dans cet article le système habituel, c'est-à-dire le nom du Codex ou manuscrit et le numéro du feuillet avec l'indication recto (r.) ou verso (v.).

\section{RÉSUMÉS}

Les dessins de machines que l'on trouve dans l'œuvre de Léonard de Vinci ne se réduisent pas à un compte rendu graphique d'inventions, les siennes et celles qu'il a pu observer mais, mis en parallèle avec l'ensemble des Carnets, ils manifestent une véritable vision du monde.

The drawings of machines, his own and those he observed, found in Leonardo da Vinci's work must not be reduced to a graphic account of inventions, but compared to the whole of the Codex, they manifest a true vision of the world.

\section{INDEX}

Mots-clés : histoire des techniques, dessin, ingénieur, moteur, mécanique, invention

Keywords : history of technology, drawing, engine, mechanics, engineer, invention 


\section{AUTEUR}

\section{GÉRARD CHAZAL}

Professeur honoraire d'histoire et philosophie des sciences de l'Université de Bourgogne. Je me suis intéressé particulièrement aux machines, depuis les plus anciennes de l'École d'Alexandrie jusqu'à l'informatique et la robotique auxquelles j'ai consacré plusieurs ouvrages. 